\title{
Modifying the microstructure and morphology of film surface layers by manipulating chemical vapor deposition reactor conditions
}

\author{
F. Paritosh \\ Department of Chemical Engineering, University of Michigan, Ann Arbor, Michigan 48109 \\ and Princeton Materials Institute and Department of Mechanical and Aerospace Engineering, \\ Princeton University, Princeton, New Jersey 08544 \\ D. J. Srolovitz ${ }^{\text {a) }}$ \\ Princeton Materials Institute and Department of Mechanical and Aerospace Engineering, \\ Princeton University, Princeton, New Jersey 08544
}

(Received 10 October 2000; accepted for publication 8 February 2001)

\begin{abstract}
Two spatial dimension front tracking simulations have been performed to study the growth of polycrystalline, faceted films from randomly oriented nuclei. We present strategies to optimize the microstructure, morphology, and texture of thin films during chemical vapor deposition. In particular, we examine how changes in reactor conditions can be used to modify the mean grain size, surface roughness, crystallographic texture, and growth zones. Changing growth conditions once the target bulk film structure is established can be used to establish a thin surface region with much different structural characteristics. Analytical models are provided to aid in choosing the appropriate changes in reactor conditions and surface layer thickness to achieve optimal properties. (C) 2001 American Institute of Physics. [DOI: 10.1063/1.1360216]
\end{abstract}

\section{INTRODUCTION}

The microstructure, morphology, and crystallographic texture of thin films play key roles in determining physical properties important for many applications, including microelectronic devices, magnetic recording media, and wear resistant coatings. ${ }^{1,2}$ Most of the microstructural, morphological, and crystallographic properties are inherited from the film growth process. Hence, control of the evolution of these structural characteristics is an important goal of thin film deposition processes. Synthesis of highly oriented polycrystalline thin diamond films by chemical vapor deposition is of special interest because of the promise it holds for both electronics and coatings applications. In faceted polycrystalline films, such as diamond, the fiber texture and morphology are critically dependent upon the growth conditions. For many applications of diamond thin film it is desirable to have large grain sizes (less scattering), a strongly textured (pseudosingle crystals) film, growth from particular facets (the point defect incorporation rate varies strongly with growth facet), and a flat surface (to minimize polishing costs). This article examines one approach that can be used to control conflicting microstructural/morphological/texture demands.

One approach to balancing microstructural and morphological requirements is to the grow the film under one set of conditions appropriate to obtain the desired microstructure and texture and then switch reactor conditions near the end of growth to modify the final surface morphology. A related approach has been used to control nucleation, wherein the film nucleates under one set of reactor conditions followed by a change in reactor conditions for the remaining film growth yielding strongly textured (nearly single crystal) dia-

${ }^{a)}$ Electronic mail: srol@ princeton.edu mond films. ${ }^{3,4}$ In a related study, microstructure control during the sputter deposition of Ta films was achieved by modifying the Ar sputter gas pressure dynamically during film growth. ${ }^{1}$ They observed that upon changing the sputter gas pressure from 15 to $1.7 \mathrm{mTorr}$ the initially random texture evolved to a very strong (002) fiber texture and the rate of grain growth decreased.

In order to clarify the mechanisms and extent to which changing reactor conditions can be used to modify film microstructure, morphology and texture, we performed a series of two-dimensional microstructure evolution (front tracking) simulations in which we discretely modify the reactor conditions during film growth. Koidl et al. ${ }^{5}$ originally introduced the concept of a growth parameter $\alpha$ (see later) that can be used to relate reactor conditions (e.g., $\mathrm{CH}_{4}$ concentration and growth temperature) to the rate of growth of particular facets ${ }^{5-9}$ Koidl et al. ${ }^{5}$ also presented some preliminary simulations that indicated how changing $\alpha$ would modify film structure. In our simulations, we grow the film with a particular value of $\alpha$ and then abruptly switch $\alpha$ at a fixed film thickness. We investigate the effects of initial and final values of $\alpha$ on the film microstructure, morphology, grain size, crystallographic texture, growth surface, and film roughness. Analysis of these results provides the requisite guidelines for designing film growth schedules to optimize film structure.

\section{SIMULATION METHOD}

The simulation algorithm is based on the principal of evolutionary selection as suggested by Kolmogorov ${ }^{10}$ and Van der Drift. ${ }^{11}$ The model consists of an array of crystallites where each facet grows normal to itself at a fixed, prescribed velocity, depending on reactor conditions. All the crystal nu- 


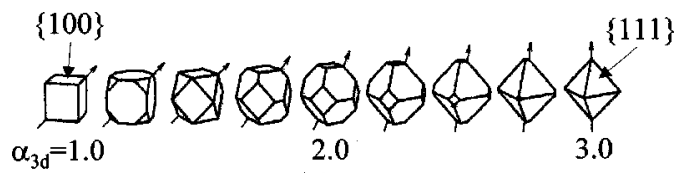

(a)

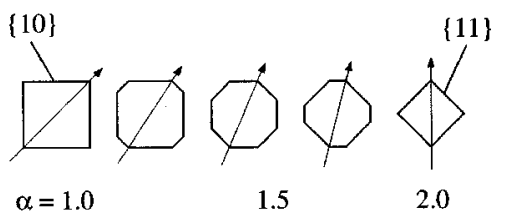

(b)

FIG. 1. Idiomorphic crystal shapes for several values of $\alpha$ in (a) three dimensions and (b) two dimensions. The arrows indicate the direction of fastest growth.

clei form simultaneously on the substrate (i.e., no renucleation occurs during growth) with random orientation with respect to the substrate. As the faceted crystallites grow, they impinge upon each other, creating a continuous, polycrystalline film. In the present model, once a corner where two facets meet disappears, it will never reappear implying that the model is truly based on local dynamics (note, this is not done properly in many simulations in the literature). A grain boundary is formed where two crystallites touch each other and it extends as the two grains meeting there continue to grow. The grain boundary location is, therefore, simply a trace in time of the point where surfaces of different grains meet (there is no grain boundary migration). Paritosh et al. ${ }^{12}$ discussed the simulation algorithm in detail. This simulation method is different from the simulations performed by Barrat et al. ${ }^{13}$ where they consider only the outer envelope of crystals growing without any restrictions.

Diamond films typically only exhibit $\{111\}$ and $\{100\}$ facets. Which of these facets dominate the structure depending on the ratio of their relative growth velocities, $\alpha_{3 d}$ $=\sqrt{3} v_{100} / v_{111},{ }^{6}$ as shown in Fig. 1(a). In the present twodimensional simulations, we focus on the 2- $d$ analog of these facets, i.e., $\{11\}$ and $\{10\}$ facets. The corresponding ratio of the facet velocities determine the growth morphology (i.e., the asymptotic shape of an isolated growing crystallite $)^{5}$

$$
\alpha_{2 d}=\sqrt{2} \frac{v_{10}}{v_{11}},
$$

where the numerical prefactor $\sqrt{2}$ in this velocity ratio is chosen such that simple, highly symmetric morphologies occur at integer values of $\alpha_{2 d}$ (i.e., $\alpha_{2 d}=1$ or 2). If such a crystal, with $\{11\}$ and $\{10\}$ facets and velocities described by $\alpha_{2 d}$, were allowed to grow without impingement, it will asymptotically approach a characteristic, idiomorphic shape, as shown in Fig. 1(b).

Each simulation begins by randomly choosing the location and orientation of the crystalline nuclei on the substrate. Each crystallite is completely described by a set of velocity vectors, which completely defines its orientation, facet velocities, and the position of its origin. A vertex, in the present two-dimensional model, is an intersection between two facets within an individual crystalline grain or where two facets from different grains meet. The vertex velocity vector is calculated from the velocities and orientation of the bounding facets (which could be either $\{11\}$ or $\{10\}$ ). While the facet orientations are fixed by the crystallographic orientation of the grain relative to the substrate, the positions and existence of individual vertices will change during the simulation. The simulation proceeds by integrating the equations of motion of the vertices forward in time. The time step used for the finite difference solution of the equation of motion for the vertex is chosen to be the time required for the next vertexvertex intersection in the entire system. Intersection times are precalculated for each vertex pair. When an intersection does occur, the facet between the intersecting vertices is removed and the velocity and orientation of the new vertex formed is determined from the orientations of the new neighboring facets.

In order to produce films of particular structural characteristics (both during growth and etching), we focus on the parameter that control the competitive growth of the different grains, i.e., $\alpha$. As described earlier, $\alpha$ can be manipulated in an experiment by changing the composition of the gas within the reactor or the reactor temperature. In the present simulations, we directly manipulate the value of $\alpha$. Changing the value of $\alpha$, changes the relative facet velocities which, in turn, changes the vertex velocities and, hence, the growth competition that is occurring.

Since the locations of the nuclei and their orientations are chosen at random, care must be used to insure statistically significant results. To this end, each simulation tracks the evolution of 1000 grains and each data point represent an average over ten simulations. All length scales are normalized by the mean spacing between the nuclei at the beginning of the simulations, $d_{0}$, and simulation times are normalized such that the velocities of all $\{11\}$ facets are fixed at unity.

\section{MICROSTRUCTURE AND MORPHOLOGY}

\section{A. Grain microstructure}

The growth conditions in the reactor determine the relative growth rate of the different crystallographic facets and through this, the grain orientation and the ratio of the roughness to grain size, as discussed in Ref. 12. These factors, in turn, determine the overall microstructure and morphology of the growing film. Figure 2(a) shows the temporal evolution of the film surface morphology and grain structure that occurs upon changing growth conditions from $\alpha_{2 d}=1.05$ to $\alpha_{2 d}=1.95$ at $h=400 d_{0}$ (i.e., where the $\alpha_{2 d}=1.05$ microstructure is well established). $\alpha_{2 d}=1.05$ and $\alpha_{2 d}=1.95$ were chosen because they are near the extremes of idiomorph shapes (see Fig. 1), while still showing both $\{11\}$ and $\{10\}$ facets. As the film thickens, the grain size increases by the faster growing grains overgrowing their slower neighbors.

The $\{11\}$ facets grow faster than their $\{10\}$ counterparts in the $\alpha_{2 d}=1.05$ film. In this case, the $\{11\}$ facets dominate the surface and are nearly parallel to the substrate, while the minority $\{10\}$ facets are at a large angle relative to the substrate normal, as described previously in Ref. 12. Upon changing $\alpha_{2 d}$ from 1.05 to 1.95 [Fig. 2(a)], the vertex velocity vectors change both in magnitude and direction (i.e., $v_{11}$ 

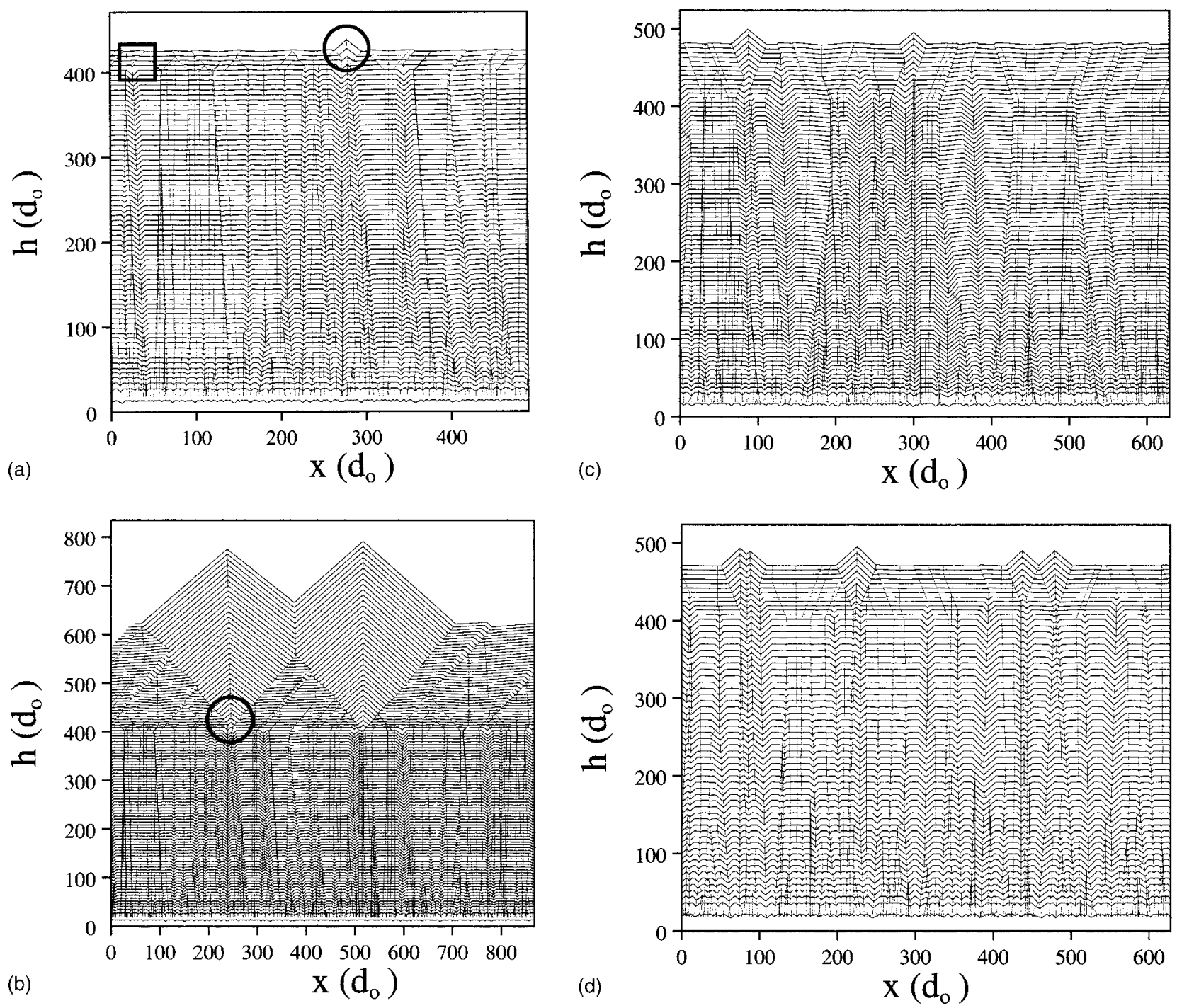

FIG. 2. The microstructures of polycrystalline diamond films for the transition from (at $h / d_{0}=400$ ) (a) and (b) $\alpha_{2 d}=1.05$ to 1.95 (shown at $h / d_{0}=450$ and 800 , respectively), and (c) $\alpha_{2 d}=1.95$ to $\sqrt{2}$, and (d) $\alpha_{2 d}=\sqrt{2}$ to 1.95 . The nearly horizontal lines correspond to the position of the growth surface at different times, while the more nearly vertical lines indicate the location of the grain boundaries. The films were all grown from 1000 randomly oriented nuclei of zero size. Only half of the film width is shown. All lengths are in units of the initial mean spacing between nuclei $\left(d_{0}\right)$.

changes from greater to less than $\left.v_{10}\right)$. Hence, the slower growing $\{11\}$ facets expand at the expense of the $\{10\}$ facets [see Fig. 3(a) and the region inside the square in Fig. 2(a)]. This expansion continues, until the $\{11\}$ facet meets another $\{10\}$ surface with which it forms a concave surface. In such a scenario, faster growing $\{10\}$ facets overgrow slower growing $\{11\}$ ones (cf. the Borgstrom construction ${ }^{14}$ for crystal growth). This process leads to a smoother, $\{11\}$-dominated surface morphology soon after $\alpha_{2 d}$ is changed. Figure 2(b) shows the continuation of the growth of the film, shown in Fig. 2(a), to later times. While the initial change from $\alpha_{2 d}$ $=1.05$ to $\alpha_{2 d}=1.95$ led to the smoothing of the film surface, continued growth produces a considerably rougher surface. This suggests that changing growth conditions and appropriately choosing how far to grow following the change can minimize the film roughness.

Examination of the rough surface in Fig. 2(b) shows that the presenting facets bounding the triangular features are predominantly the $\{10\}$ facets that make a nearly $45^{\circ}$ angle with

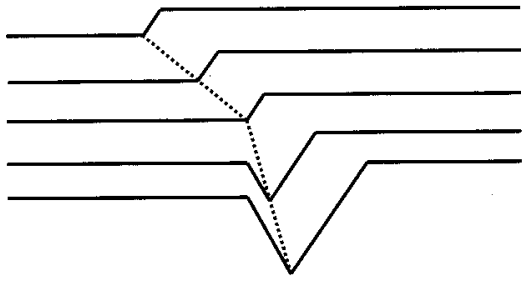

(a)

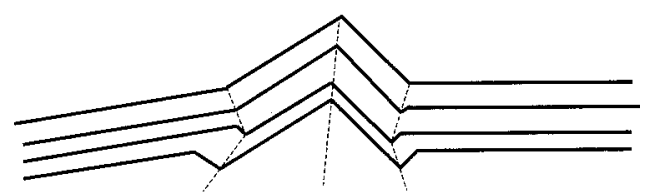

(b)

FIG. 3. Schematic illustration of two features in the surface evolution: (a) surface smoothening associated with the disappearance of a concave surface feature and (b) surface roughening associated with the enlarging of a convex surface feature. 
respect to the substrate normal [see Fig. 3(b) and the circled regions of Figs. 2(a) and 2(b)]. A grain boundary is found at the center of each of these triangular features. Tracing each of these grain boundaries back into the $\alpha_{2 d}=1.05$ section of the film (i.e., before $\alpha_{2 d}$ is changed) shows that these straight, nearly vertical grain boundaries continue through the $\alpha_{2 d}=1.95$ section of the film right into the $\alpha_{2 d}=1.05$ region. Such straight, nearly vertical boundaries only occur if the two grains that meet at the boundary have almost exactly the same crystallographic orientation. The grains that make up the triangular features that dominate the microstructure at late times are the few $\langle 11\rangle$ oriented grains that survived the $\langle 10\rangle$ texture selection that occurred when $\alpha_{2 d}$ was 1.05 . The change in the apparent roughness following the texture change is dominated by the degree to which nearly $\langle 11\rangle$ oriented grains survived the initial growth.

Figures 2(c) and 2(d) show the evolution of the film microstructure and morphology following a change from $\alpha_{2 d}=1.95$ to $\sqrt{2}$ and $\alpha_{2 d}=\sqrt{2}$ to 1.95 , respectively. $\alpha_{2 d}$ $=\sqrt{2}$ corresponds to equal size $\{11\}$ and $\{10\}$ facets in the idiomorphs of Fig. 1. Both microstructures show similar features, such as an abrupt change in grain boundary inclination and surface morphology following the change in $\alpha_{2 d}$. Similar features were observed in Figs. 2(a) and 2(b), where $\alpha_{2 d}=1.05$ is changed to $\alpha_{2 d}=1.95$. Comparisons of the microstructures for the different changes in $\alpha_{2 d}$ (Fig. 2) demonstrates that the magnitude of the change in boundary inclination following the change in $\alpha_{2 d}$ increases as the magnitude by which $\alpha_{2 d}$ is changed increases.

The change in slope of the grain boundaries upon change in $\alpha_{2 d}$ may be simply understood by consideration of the initial microstructure and the idiomorphs of Fig. 1. In the microstructure immediately prior to the change in $\alpha_{2 d}$, most of the grains have nearly the same orientation with respect to the substrate, as described in Ref. 12. This orientation is such that the fastest growing direction is oriented normal to the substrate; namely

$$
\phi=\cot ^{-1}\left(\frac{\alpha_{2 d}}{2-\alpha_{2 d}}\right),
$$

where $\phi$ is the angle between the fastest growing direction and the [01] direction. Two grains with nearly the same orientation will be separated by grain orientations, which are nearly parallel, and grain boundaries that are nearly normal to the substrate. If the facets that meet at the grain boundary are of different type, a change in $\alpha_{2 d}$ will produce an immediate change in the grain boundary trajectory (i.e., the angle the grain boundary makes with respect to the substrate normal, $\theta$ ). On the other hand, if the facets that meet at the grain boundary are identical, changing $\alpha_{2 d}$ has no impact on $\theta$. The change in grain boundary angle corresponds to the change in the fastest growing direction. Hence, a change in $\alpha_{2 d}$ results in a change in the mean orientation of the grain boundaries with respect to the substrate normal (i.e., the mean value of $|\Delta \theta|$ ). Therefore, the change in $\theta$ may be determined through Eq. (2) as

$$
|\overline{\Delta \theta}|=\frac{1}{2}\left|\tan ^{-1}\left(\frac{\alpha_{2 d}^{+}}{2-\alpha_{2 d}^{+}}\right)-\tan ^{-1}\left(\frac{\alpha_{2 d}^{-}}{2-\alpha_{2 d}^{-}}\right)\right|,
$$

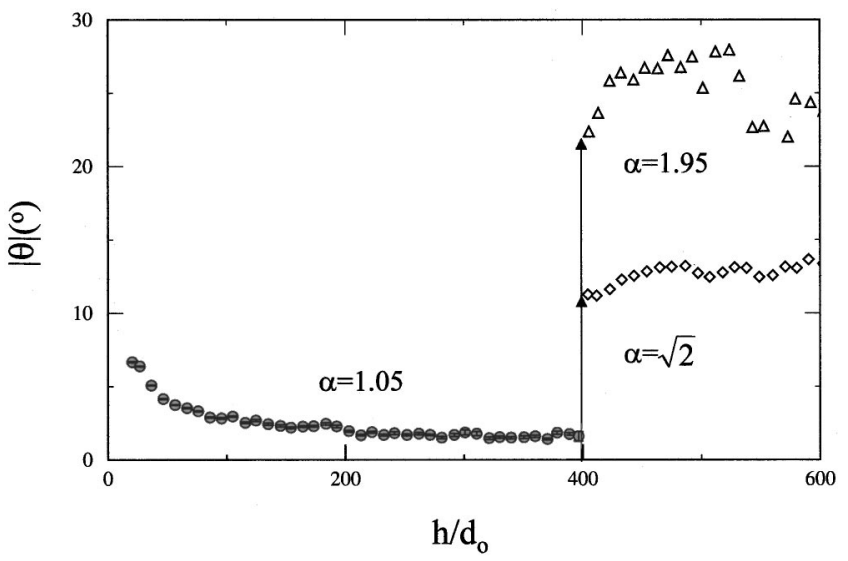

FIG. 4. Mean grain boundary orientation deviation from the substrate normal $|\theta|$ when a film grown with $\alpha_{2 d}=1.05$ is subjected to growth conditions where $\alpha_{2 d}=1.95$ and $\sqrt{2}$.

where the superscripts "+" and "-" indicate the values following and prior to the change in $\alpha_{2 d}$. The factor of $\frac{1}{2}$ in Eq. (3) accounts for the observation that in nearly $50 \%$ of the cases, the two facets that meet at a grain boundary are of the same type (i.e., $\{11\}$ or $\{10\}$ ). This is consistent with the change in boundary orientations seen in Fig. 2 (i.e., $|\Delta \theta|$ $=\pi / 4$ for $\alpha_{2 d}=1$ changing to $\alpha_{2 d}=2$ and $|\Delta \theta|=\pi / 8$ for $\alpha_{2 d}=2$ changing to $\sqrt{2}$ or $\sqrt{2}$ changing to 2 ).

Figure 4 shows the relationship between the change in the mean boundary inclination $|\theta|$ and the film thickness for the cases of $\alpha_{2 d}=1.05$ changing to 1.95 and $\sqrt{2}$. In the initial film $\left(\alpha_{2 d}=1.05\right)$, the grain boundaries become increasingly normal to the substrate $(|\theta|$ decreases $)$ as the film grows and the texture sharpens. Upon changing $\alpha_{2 d},|\theta|$ undergoes a sharp jump, indicating that the mean boundary inclination instantaneously changes. This can be seen directly in the microstructures shown in Fig. 2. $|\overline{\Delta \theta}| \approx 21^{\circ}$ when $\alpha_{2 d}$ changes from 1.05 to 1.95 and $|\overline{\Delta \theta}| \approx 11^{\circ}$ when $\alpha_{2 d}$ changes from 1.05 to $\sqrt{2}$. The corresponding values predicted on the basis of the idiomorphs in Eq. (3) are $20.3^{\circ}$ and $9.9^{\circ}$, respectively. The agreement between the simulations and the predictions is well within the statistical error of the simulation results.

\section{B. Grain size evolution}

The evolution of the mean grain size $\bar{d}$ with film thickness $h$ is shown in Fig. 5(a) for simulations in which $\alpha_{2 d}$ $=1.05$ is changed to $1.95, \sqrt{2}$, or 1.20 . Clearly, the growth rate increases abruptly for all changes in $\alpha_{2 d}$. It was shown in Refs. 12 and 15 that the mean grain size increases as a parabolic function of film thickness at fixed $\alpha_{2 d}$ :

$$
\bar{d}=A h^{\beta},
$$

where $\beta=1 / 2$ and $A$ is a constant. This is consistent with the slope in Fig. 5(a) observed prior to the change in $\alpha_{2 d}$, as shown in Table I [the exponent $\beta$ measured from Fig. 5(a) is 0.47 , which is slightly smaller than the predicted value]. The slight deviation of $\beta$ from $1 / 2$ prior to the change in $\alpha_{2 d}$ would disappear if the simulation were run to much longer times/larger thickness. Following the change in $\alpha_{2 d}$, the ini- 

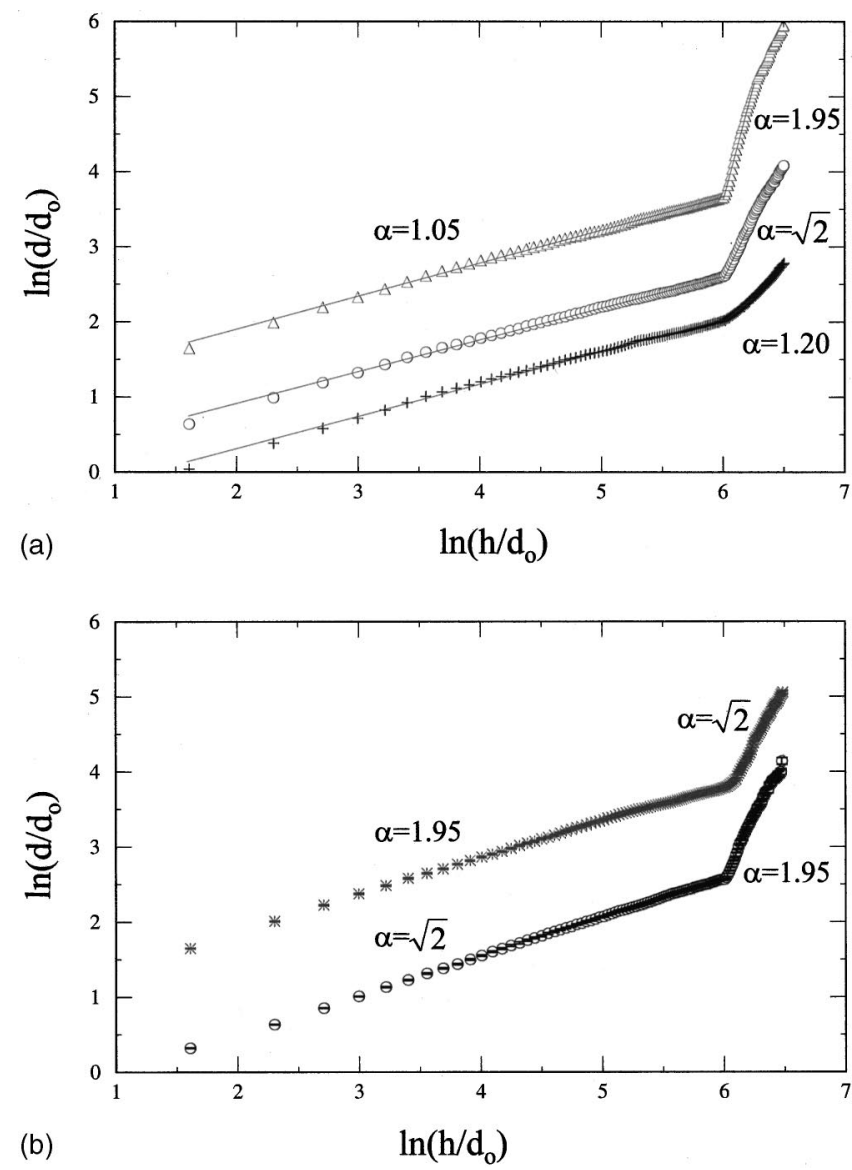

FIG. 5. Variation of the mean grain size, $\bar{d} / d_{0}$, with film thickness, $h / d_{0}$, for a change in growth conditions (a) from $\alpha_{2 d}=1.05$ to $1.20, \sqrt{2}$ and 1.95 and (b) from $\alpha_{2 d}=1.95$ to $\sqrt{2}$ and $\alpha_{2 d}=\sqrt{2}$ to 1.95 .

tial slope (i.e., $d \ln \bar{d} / d \ln h$ ) in Fig. 5(a) increases abruptly by an amount that depends on $\alpha_{2 d}$ (see Table I). This is consistent with the observations and discussion of Fig. 2 presented earlier. The change in $\alpha_{2 d}$ results in a rotation of the grain boundaries such as to increase the angle they make with the substrate normal (i.e., an increase in $|\bar{\theta}|$ ). The magnitude of this rotation increases with increasing change in $\alpha_{2 d}$.

As $|\bar{\theta}|$ increases, the time required for two adjacent grain boundaries to intersect decreases (on average). Since it is these intersections that pinch off grains, the rate of grain size evolution increases with increasing $\alpha_{2 d}$. A simple analysis shows that the rate of change of the grain size $d(\bar{d}) / d h$ should be directly proportional to the tangent of the angle the grain boundaries make with the normal, i.e., $d(\bar{d}) / d h$ $\sim \tan (|\overline{\Delta \theta}|)$. We can extract the value of $|\overline{\Delta \theta}|$ from the expression describing the grain boundary orientation in terms

TABLE I. Comparison of slope (exponent $\beta$ ) prior to $\alpha_{2 d}$ change and immediately following change in $\alpha_{2 d}$.

\begin{tabular}{lcc}
\hline \hline & $\beta$ & $\beta$ \\
& (prior to $\alpha_{2 d}$ change) & (following $\alpha_{2 d}$ change) \\
\hline$\alpha_{2 d}=1.05$ to $\alpha_{2 d}=1.20$ & 0.47 & 1.2 \\
$\alpha_{2 d}=1.05$ to $\alpha_{2 d}=\sqrt{2}$ & 0.47 & 3.4 \\
$\alpha_{2 d}=1.05$ to $\alpha_{2 d}=1.95$ & 0.47 & 6.4 \\
\hline \hline
\end{tabular}

of the idiomorphs [Eq. (3)]. The exponent $\beta^{+}$extracted from Fig. 5(a) is defined as $d(\ln \bar{d}) / d(\ln h)=(h / \bar{d})[d(\bar{d}) / d h]=\beta$. Since the values of $h / \bar{d}$ are exactly the same for all three curves in Fig. 4(a) at the point where $\alpha_{2 d}$ is changed, $d(\bar{d}) / d h$ is proportional to $\beta^{+}$. The values of $\beta^{+}$from Table I vary in the proportions $0.19: 0.53: 1$ for $\alpha_{2 d}^{+}=1.20, \sqrt{2}$ and 1.95. The values of $\tan (|\overline{\Delta \theta}|)$, determined from Eq. (3) vary in the proportions 0.17:0.42:1 for the same values of $\alpha_{2 d}^{+}$. Clearly, the rate of change of the grain size $d(\bar{d}) / d h$ determined from the simulations is in reasonable agreement with the prediction $d(\bar{d}) / d h \sim \tan (|\overline{\Delta \theta}|)$, where $(|\overline{\Delta \theta}|$ is determined from the idiomorph expression, Eq. (3).

We performed additional simulations designed to validate the concept that grain size evolution is controlled by changes in mean grain boundary angle. In the first test, we ran a simulation where we changed $\alpha_{2 d}$ from $\sqrt{2}$ to 1.95 and then ran another simulation where we changed $\alpha_{2 d}$ from 1.95 to $\sqrt{2}$. The data are shown in Fig. 5(b) and show that $\beta^{+}$for the simulations are identical to within the simulation error $\left(\beta^{+}=3.7\right.$ and 3.3 , respectively). This shows that reversing the change in $\alpha_{2 d}$ (which does not change the grain boundary angles) does not change $\beta^{+}$. Similarly, choosing pairs of values of $\alpha_{2 d}$ that correspond to the same values of $\tan (|\overline{\Delta \theta}|)$ [as per Eq. (3)], yield the same values of $\beta^{+}$.

\section{Roughness}

Examination of the microstructures in Fig. 2 suggests that the change in the relative growth rates of facets $\left(\alpha_{2 d}\right)$ could be exploited to modify the roughness of the film. The effects of changes in $\alpha_{2 d}$ on the rms roughness of the growth surface, $w$, is shown in Fig. 6(a) for different $\alpha_{2 d}$ transitions. Prior to the transition, the roughness grows with the film thickness according to the relationship

$$
w=B h^{\gamma},
$$

where $B$ is a constant and the exponent $\gamma=0.5 \pm 0.2{ }^{12}$ This exponent is the same as that describing the evolution of the grain size. This is not surprising since most grains exhibit two facets between grain boundaries such that, for example, doubling the grain size simply linearly scales the shape of the faceted grain surface.

Figure 6(a) shows that upon changing $\alpha_{2 d}$ (from 1.05 to $1.20, \sqrt{2}$ and 1.95), the rms roughness $w / d_{0}$, initially drops by nearly a factor of 2 before rising again [see inset in Fig. 6(a)]. This implies that it is possible to decrease the roughness of a film by changing growth conditions. These data also show that there is an ideal thickness corresponding to a minimum roughness. Figure 6(b) shows two additional cases, corresponding to changing $\alpha_{2 d}$ from 1.95 to $\sqrt{2}$ and from $\sqrt{2}$ to 1.95 . In the former case, a minimum in the film roughness occurs upon changing $\alpha_{2 d}$, but in the latter case the roughness increases monotonically with thickness following the change in $\alpha_{2 d}$.

In order to understand why minima in the roughness occur following changes in $\alpha_{2 d}$ in some cases and not in others, we consider how the roughness varies with $\alpha_{2 d}$ in a film grown at fixed $\alpha_{2 d}$. The variation of the ratio of the rms roughness to mean grain size $(w / \bar{d})$ is shown in Fig. 7 for 


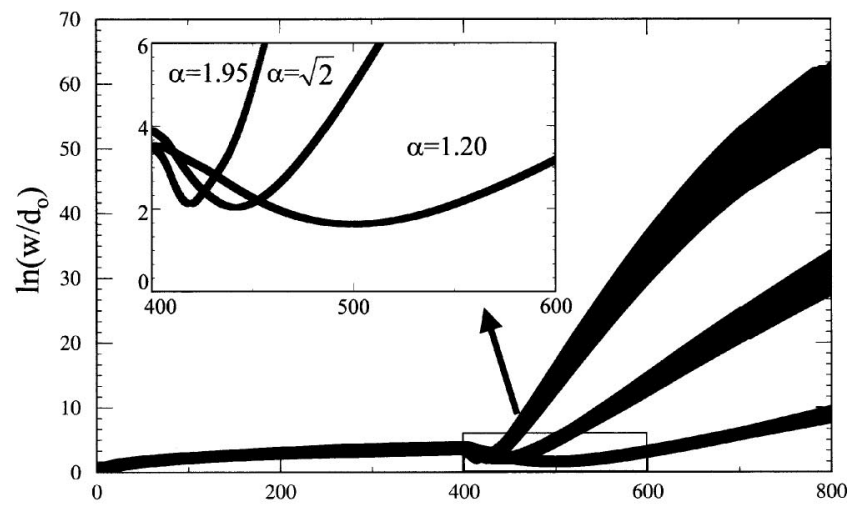

(a)

$\ln \left(\mathrm{h} / \mathrm{d}_{\mathrm{o}}\right)$
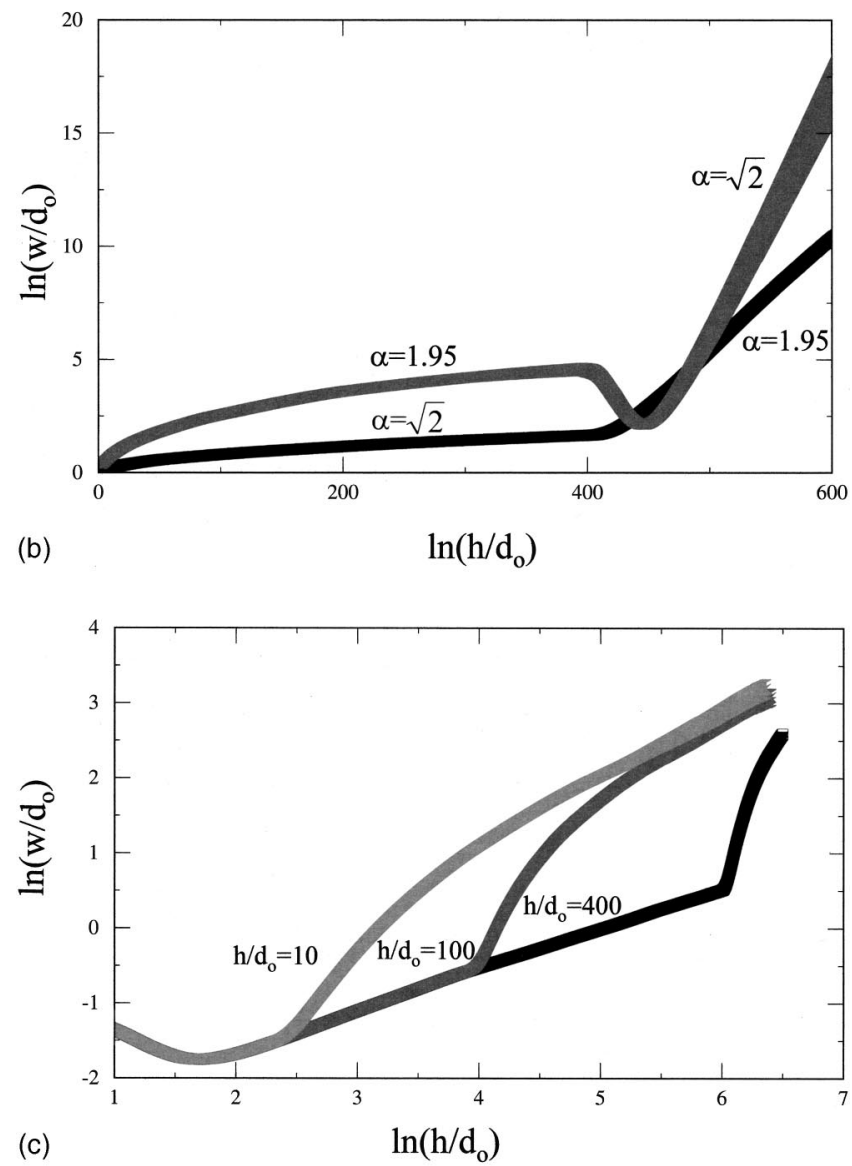

FIG. 6. Variation of the root mean square roughness, $w / d_{0}$, with film thickness, $h / d_{0}$ (a) for transitions from $\alpha_{2 d}=1.05$ to $1.20, \sqrt{2}$, and 1.95 at $h / d_{0}=400$, (b) for transitions from $\alpha_{2 d}=1.95$ to $\sqrt{2}$, and $\alpha_{2 d}=\sqrt{2}$ to 1.95 at $h / d_{0}=400$, and (c) for transitions from $\alpha_{2 d}=\sqrt{2}$ to 1.95 at $h / d_{0}=10$, 100 , and 400 .

films grown under constant $\alpha_{2 d}$ conditions. The roughness shows equal maxima at $\alpha=1$ and $\alpha=2$ and monotonically decays to a minimum at $\alpha=\sqrt{2}$. The continuous curve in Fig. 7 was derived from the idiomorph shape (Fig. 1) by orienting the idiomorph such that the fastest growing direction is normal to the substrate (i.e., one of the corners corresponds to the highest point on the surface of a grain) and assuming that the grain boundaries are at the positions where the other corners bounding the facets meet at this peak

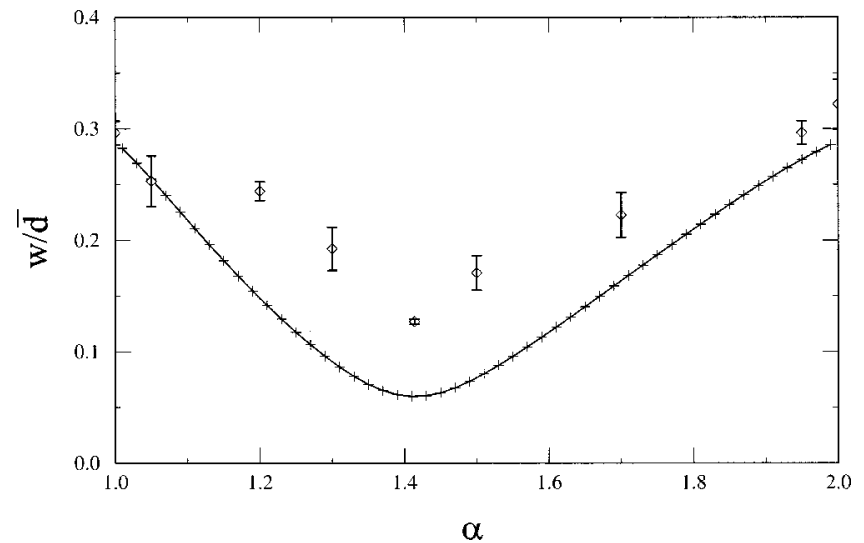

FIG. 7. The ratio of the film roughness to the grain size vs $\alpha_{2 d}$, for simulations performed at constant $\alpha_{2 d}$, measured at late time. The solid line and + symbols represents the analytical prediction from Eq. (6) and the simulation data are represented by the diamonds.

$$
\left(\frac{w}{d}\right)^{2}=\frac{m_{a}^{2} L_{a}^{3}+m_{b}^{2} L_{b}^{3}}{3\left(L_{a}+L_{b}\right)^{3}}-\left[\frac{L_{a}+L_{b}-2 L_{a} L_{b}}{2\left(L_{a}+L_{b}\right)^{2}}\right]^{2},
$$

where $\quad L_{a}=\sin (2 \phi), \quad L_{b}=\cos (2 \phi), \quad m_{a}=\tan (\phi), \quad b m_{b}$ $=[\tan (\phi)-1] /[\tan (\phi)+1]$, and $\phi$ is related to $\alpha$ as in Eq. (2). The analytical expression captures the overall behavior of the dependence of the roughness of the microstructures on $\alpha$, including the roughness and $\alpha$ values where the maxima occur and the $\alpha$ value corresponding to the minimum roughness.

We now return to the question of why the roughness shows a minimum with increasing $h$ for some changes in $\alpha_{2 d}$ and not in others. We invariably find [Figs. 6(a) and 6(b)] that if the steady-state roughness corresponding to the initial value of $\alpha_{2 d}$ [Fig. 7, Eq. (6)] is greater than the steady-state roughness corresponding to the new value of $\alpha_{2 d}$, then the roughness versus $h$ plots corresponding to a change in $\alpha_{2 d}$ exhibit minima. Additional simulations show that the roughness also decreases if the initial and final values of $\alpha_{2 d}$ bracket (are on different sides of) the minimum. If these condition fail, then no minimum occurs, as shown in Fig. 6(b).

Figure 6(c) shows the effect of growing the film to different thickness prior to changing $\alpha_{2 d}$ from $\sqrt{2}$ to 1.95 (where no minima in the roughness occur). Following a change in $\alpha_{2 d}$, the surface morphology undergoes a transient prior to settling back into normal growth-defined here as a return to the $d w / d h$ dependence suggested by Eq. (5) with $\gamma=1 / 2$. Figure 6(c) shows that the duration of this transient depends on the thickness at which $\alpha_{2 d}$ is changed.

If a minimum in the roughness exists, then it is useful to know how long to grow, following the change in $\alpha_{2 d}$, to reach that minimum roughness. As described earlier, the change in the microstructure upon changing $\alpha_{2 d}$ is associated with the change in angles the grain boundaries make with respect to the normal. This change in angle may be obtained directly from Eq. (3), before. If two adjacent grain boundaries turn toward each other, they impinge after they grow by an amount equal to the product of half the grain size $d$ and the boundary slope, where the latter is simply 
TABLE II. Comparison of the film thickness at which the roughness is a minimum according to Fig. 6(a) (simulation) and Eq. (7).

\begin{tabular}{lcc}
\hline \hline & $\frac{\Delta h}{d_{0}}$ (simulation) & $\frac{\Delta h}{d_{0}}$ [Eq. (7)] \\
\hline$\alpha_{2 d}=1.05$ to $\alpha_{2 d}=1.20$ & 100 & 91 \\
$\alpha_{2 d}=1.05$ to $\alpha_{2 d}=\sqrt{2}$ & 41 & 39 \\
$\alpha_{2 d}=1.05$ to $\alpha_{2 d}=1.95$ & 18 & 10 \\
$\alpha_{2 d}=1.95$ to $\alpha_{2 d}=\sqrt{2}$ & 43 & 36 \\
\hline \hline
\end{tabular}

$1 / \tan (|\overline{\Delta \theta}|)$. Therefore, the amount of additional growth required to reach the minimum (if one exists) is

$$
\Delta h=\frac{\bar{d}}{2 \tan (|\overline{\Delta \theta}|)},
$$

where $\bar{d}$ is the mean grain size before the change in $\alpha_{2 d}$ $(\bar{d}=13.4$ for the data in Fig. 2). The predictions of Eq. (7) together with the positions of the minima in $w / d$ from Fig. 6 are shown in Table II. Equation (7) is in overall good agreement with the simulation data, but slightly underestimates the value of $\Delta h$ at which the minimum roughness occurs. This discrepancy is largest when $\Delta h$ is small and is associated with the error in approximating the shape of the $w$ vs $h$ curve following the change in $\alpha_{2 d}$ with a straight line.

\section{Growth zones}

The microstructures shown in Fig. 2 indicate that a fraction of the film grew from $\{11\}$ surfaces and the remaining part grew from $\{10\}$ surfaces. When $\alpha$ is changed, these relative fractions shift. Commonly, the concentration of defects incorporated within a film depends on from which surface the material grew. ${ }^{16}$ Such defects include microtwins, impurities, and vacancies. ${ }^{17}$ Therefore, the defect concentrations within the film are functions of the $\alpha$ history the film experienced during growth. Figure 8(a) shows the fraction of the film grown from $\{11\}$ and $\{10\}$ surfaces $\left(f_{\{11\}}\right.$ and $f_{\{10\}}$, respectively) as a function of film thickness for the case in which $\alpha$ is changed from $\sqrt{2}$ to 1.95 . The fraction of the entire film grown from each type of surface is simply the average of these fractions over the entire thickness.

Based upon the idiomorphs [Fig. 1(b)], we expect that for $\alpha_{2 d}=\sqrt{2}$, facets will equally likely be of $\{11\}$ and $\{10\}$ types and the fraction grown from each facet type will be equal. This is consistent with Fig. 8(a). Upon changing $\alpha_{2 d}$ to 1.95 , we observe that the fraction grown from $\{11\}$ facets increases quickly at the expense of that from $\{10\}$ facets. This is unexpected since a film grown at a constant $\alpha_{2 d}$ $=1.95$ is dominated by $\{10\}$ facets, as shown and discussed in Ref. 12. We can understand this result by considering the types of facets that meet at grain boundaries (Fig. 9). If two adjacent grains have very similar orientations and two unlike facets meet at the grain boundary, when $\alpha$ changes, there is no net change in the length of the two facets meeting at the boundary [see Fig. 9(a)]. This figure shows that at interior corners the faster growing facet consumes the slower growing facet and at exterior corners the slower growing facet preferentially lengthens leaving no net change in the relative size of the intervening facet. On the other hand, if like facets
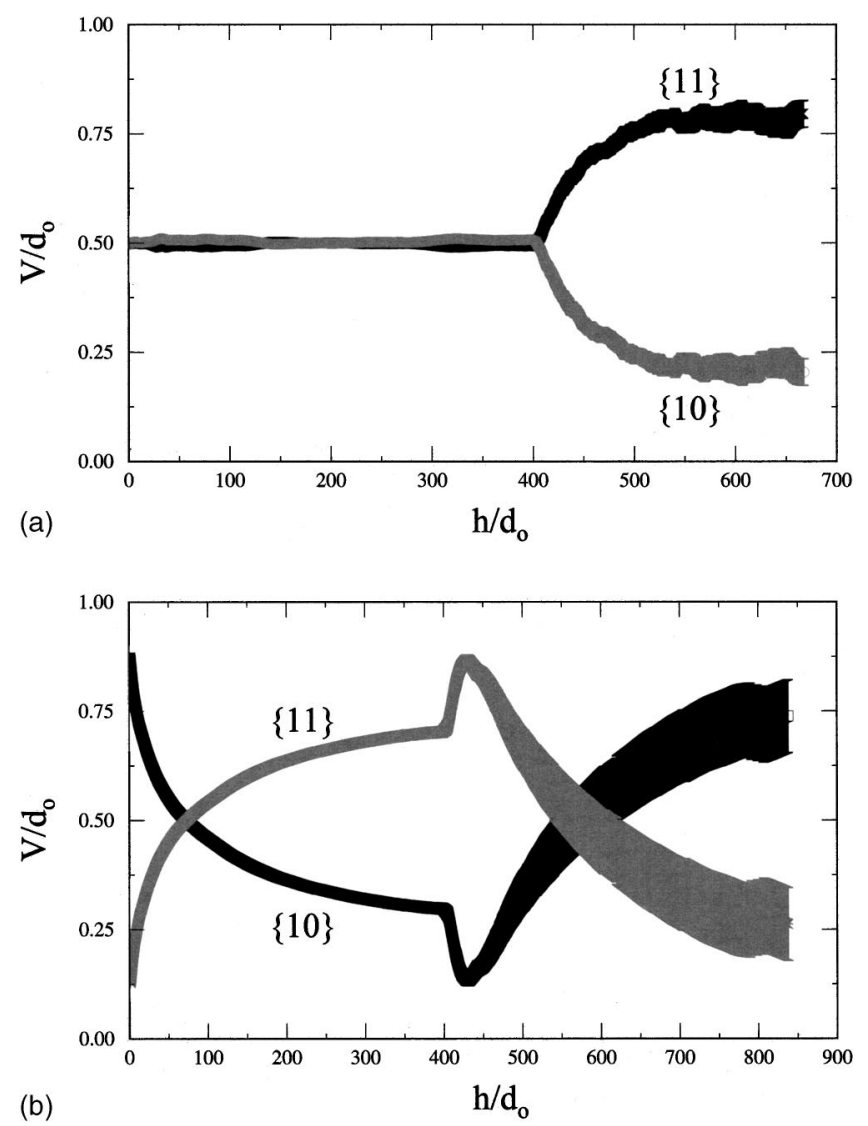

FIG. 8. Volume fraction of the film grown from $\{10\}$ (gray) and $\{11\}$ (black) facets as a function of film thickness, $h / d_{0}$, for (a) transition from $\alpha_{2 d}$ $=\sqrt{2}$ to 1.95 , and (b) transition from $\alpha_{2 d}=1.05$ to 1.95 .

meet at the grain boundaries (i.e., interior corners), the length of slower growing facets always increase at the expense of the faster growing facets [see Figs. 9(b) and 9(c)]. Figure 9(b) shows that when like facets meet at interior corners are fast growing, the valley where they meet disappears. On the other hand, Fig. 9(c) shows that if the facets that meet at an interior corner are slow growing, the valley between them gets deeper.

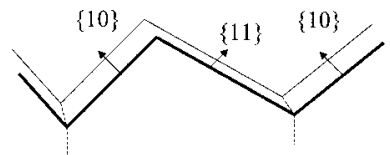

(a)

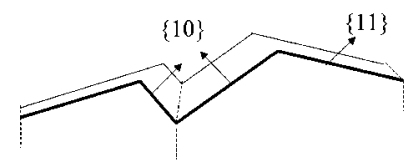

(b)

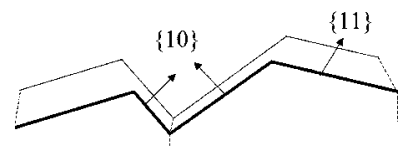

(c)

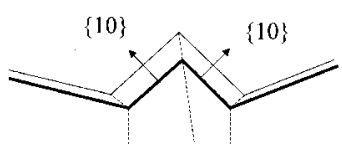

(d)
FIG. 9. Schematic illustration of surface evolution upon changes in $\alpha$. (a) unlike facets meeting at a grain boundary (dashed line) valley do not change length upon change in $\alpha$. Like facets meeting at a grain boundary will either shorten or lengthen depending on whether they grow (b) faster or (c) slower than the facets of the other type. (d) If two fast growing surfaces form an exterior corner their length increases and they dominate the morphology. 
Figure 8(b) shows the evolution of the fraction grown from the two facet types in a simulation in which $\alpha_{2 d}$ is changed from 1.05 to 1.95 . Initially, the fraction of $\{10\}$ facets is high compared to $\{11\}$ facets. This is consistent with the $\alpha_{2 d}=1.05$ idiomorph for the individual grains prior to impingement (i.e., before the film is continuous). Upon impingement, the fraction of $\{11\}$ facets rapidly increases, as discussed in Ref. 12. When $\alpha_{2 d}$ is changed to 1.95 (at $\left.h / d_{0}=400\right)$, the fraction of $\{11\}$ facets increases rapidly and then starts to slowly decrease [Fig. 8(b)]. The initial rise in the $\{11\}$ fraction following the change in $\alpha_{2 d}$ is explained in Figs. 9(b) and 9(c). The subsequent decrease of the $\{11\}$ fraction and increase of the $\{10\}$ fraction can be understood with the aid of Fig. 9(d). In short, two fast facets meeting at a grain boundary in an exterior corner [see Fig. 9(d) and the circled region of Fig. 2(a)] grow out as a triangular protrusion and eventually dominate the surface [Fig. 2(b)].

These results and analysis suggest that there are three types of behavior that can occur. The first, and special, case occurs when the initial $\alpha_{2 d}=\sqrt{2}$ such that all of the facets are equivalent. In this case, the fraction grown from $\{11\}$ and $\{10\}$ are exactly the same prior to the change in $\alpha_{2 d}$ followed by a monotonic increase in the fraction grown from the slower facets [Fig. 8(a)]. The second case, corresponds to the situation in which $\alpha_{2 d}$ is changed from below $\sqrt{2}$ to above it (or vice versa) and the behavior is that shown in Fig. 8(b) (an initial crossing of the curves for the two facets before the change in $\alpha_{2 d}$, followed by nonmonotonic behavior and a second crossing following the change in $\alpha_{2 d}$ ). The third possibility, not shown before, is when $\alpha_{2 d}$ increases from above $\sqrt{2}$ to larger values (or decreases from below $\sqrt{2}$ ). In this case, the behavior will be similar to Fig. 8(b) but without exhibiting the sharp minima or maxima shortly after the change in $\alpha_{2 d}$.

\section{CRYSTALLOGRAPHIC TEXTURE}

At the beginning of each simulation, infinitesimal crystallites are placed onto the substrate at random positions and with random orientation, yielding a flat distribution of crystal orientations. We define the orientation distribution function $P(\theta)$ as the probability that the $\langle 10\rangle$ direction of a grain makes an angle $\theta$ with respect to the substrate normal (because of the symmetry of the crystal, we restrict these angles to the range $\left.|\theta| \leqslant 45^{\circ}\right)$. We discussed the evolution of texture during film growth at constant $\alpha_{2 d}$ in Ref. 12. In short, we found that the texture sharpened during film growth with the dominant texture component consistent with the direction of the fastest growing point on the idiomorph (i.e., the arrows in Fig. 1). Since the fastest growing direction changes with $\alpha_{2 d}$, the film texture is a function of $\alpha_{2 d}$. At the film thickness where we change $\alpha_{2 d}$ in most of the present study, the crystallographic texture is already well developed and very few misoriented grains remain (i.e., with orientations more than a few degrees away from that predicted by the fastest growing direction in the idiomorph).

Figure 10 shows the evolution of film texture as a function of film thickness (starting below where the transition occurs and extending well beyond it) for the cases in which
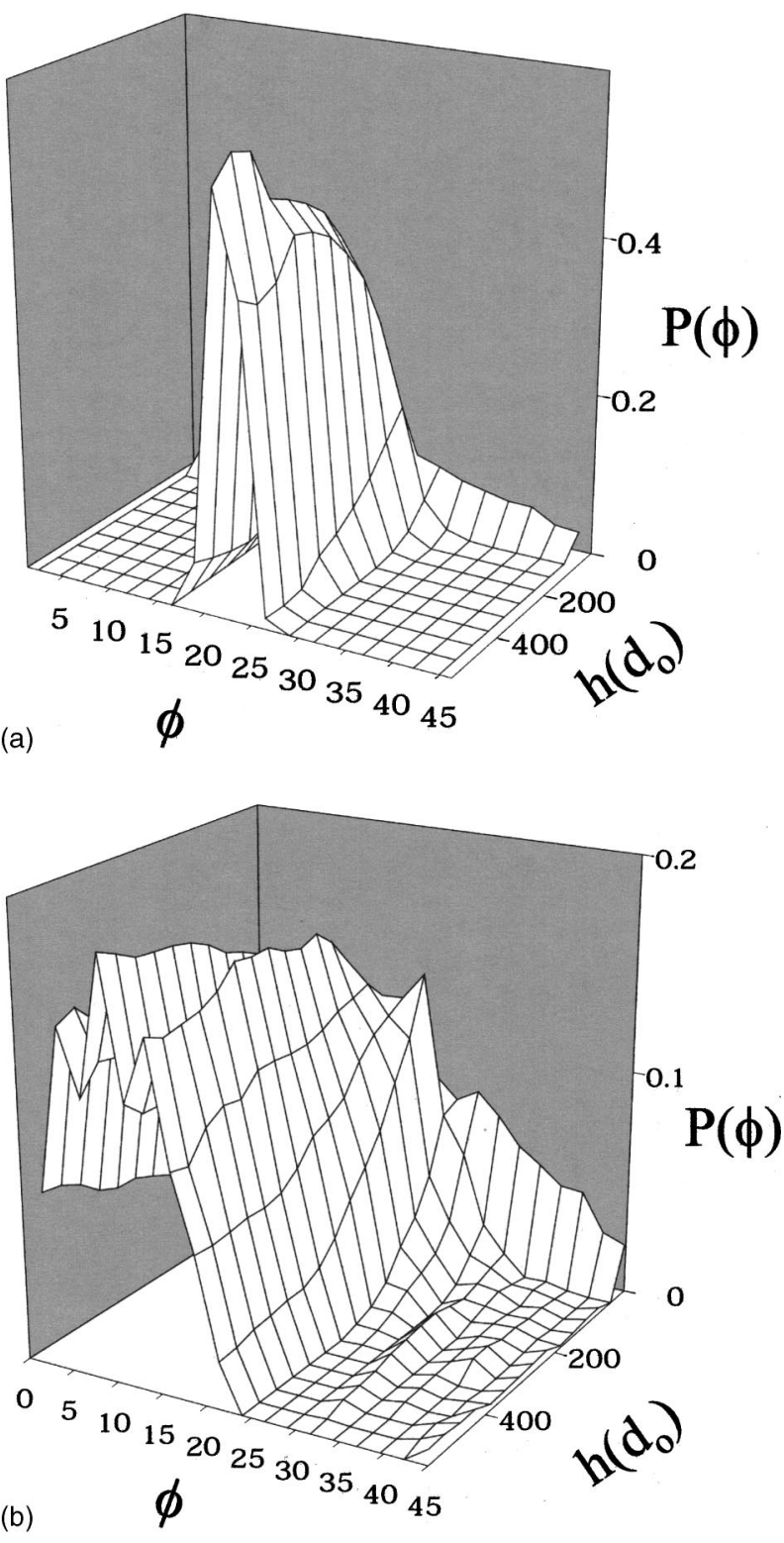

FIG. 10. Orientation distribution function $P(\phi)$ as a function of film thickness, $h / d_{0}$ for transition from: (a) $\alpha_{2 d}=\sqrt{2}$ to 1.95 (at $h / d_{0}=400$ ), (b) $\alpha_{2 d}=\sqrt{2}$ to 1.95 (at $h / d_{0}=5$ ).

$\alpha_{2 d}$ changes from $\sqrt{2}$ to 1.95 at $h / d_{0}=400$ and at $h / d_{0}=5$. In the first case [Fig. 10(a)], the change in $\alpha_{2 d}$ leads to a sharpening of the texture, but very little change in the peak angle. While we would normally expect the peak position at $\alpha_{2 d}=1.95$ to be $\phi=1.5^{\circ}$ [see Eq. (2)], no grains with orientations $\theta<15^{\circ}$ remain at the film thickness where the change in $\alpha_{2 d}$ occurred. This is because in simulations with relatively few initial grains $\left(10^{3}\right)$, there are few grains in the tails of the grain orientation distribution when the texture is already sharp and most of the grains have already disappeared. Nonetheless, close inspection of the contour lines show that the orientation distribution does exhibit a slight shift to lower values of $\phi$ following the transition. When $\alpha_{2 d}$ is changed at very small film thickness, such as $h / d_{0}=5$ [Fig. 10(b)], the orientation distribution is very broad and the peak position is clearly shifted to smaller values of $\phi$. In this case, the peak position at the end of the simulation $\left(\sim 10^{\circ}\right)$ is 
significantly closer to the predicted value of $\phi=1.5^{\circ}$ for $\alpha_{2 d}=1.95$. This pronounced difference in the two cases can be attributed to the relative sharpness of the distributions prior to the change in $\alpha_{2 d}$.

\section{CONCLUSION}

We have performed a series of simulations to examine how changing growth conditions can be used to modify the microstructure, morphology, and texture of growing films. The simulations track the evolution of the two-dimensional, faceted growth front during the deposition of polycrystalline films. This approach could be applied to optimizing the quality of diamond thin films during chemical vapor deposition. Since the microstructure, morphology, and texture evolution are sensitive to growth conditions, changing $\alpha$ during deposition provides an additional level of control not available under constant growth conditions. A possible growth scenario is to choose a value of the growth parameter $\alpha$ that yields the desired bulk texture and grain size, then changing $\alpha$ and continuing to grow to a slightly larger thickness to produce a flatter surface, a larger grain size and/or a new crystallographic texture at the surface. While the change in grain size and surface roughness occurs rapidly on changing $\alpha$, the evolution of the texture is more gradual. Following the change in $\alpha$, there is an optimal additional film thickness for which the surface roughness is a minimum. Therefore, structure optimization requires not only choosing an appropriate new value of $\alpha$ but also when to stop growth. The present simulation results and analysis provide guidelines on how to choose the optimal growth conditions and how long to grow. This same approach can be used to modify the incorporation rate of point defects and twins during growth simply by modifying the dominant growth morphology (growth facet).
Although the present simulation procedure is based on several important simplifying assumptions (two dimensions, constant velocity facet velocities at fixed reactor conditions, instantaneous changes in reactor conditions, no twinning, etc.) it does provide the insight and theoretical foundation necessary to intelligently design the film growth strategies necessary to optimize film growth. The same type of simulations could also be used to predict the evolution of morphology under etching rather than growth conditions.

${ }^{1}$ J. F. Whitacare, S. M. Yalisove, and J. C. Billelo, Mater. Res. Soc. Symp. Proc. 562, 141 (1999).

${ }^{2}$ R. M. Bradley, J. M. E. Harper, and D. A. Smith, J. Appl. Phys. 60, 4160 (1986).

${ }^{3}$ S. D. Wolter, B. R. Stoner, J. T. Glass, P. J. Ellis, D. S. Buhaenko, C. E. Jenkins, and P. Southwaorth, Appl. Phys. Lett. 62, 1215 (1993).

${ }^{4}$ B. R. Stoner, S. R. Sahaida, J. P. Bade, P. Southworth, and P. J. Ellis, J. Mater. Res. 8, 1334 (1993).

${ }^{5}$ C. Wild, N. Herres, and P. Koidl, J. Appl. Phys. 68, 973 (1990).

${ }^{6}$ C. Wild et al. Diamond Relat. Mater. 158, 158 (1993).

${ }^{7}$ B. V. Spitsyn, L. L. Bouilov, and B. V. Derjaguin, J. Cryst. Growth 52, 219 (1981).

${ }^{8}$ R. E. Clausing, L. Heatherly, L. L. Horton, E. D. Specht, G. M. Begun, and Z. I. Wang, Diamond Relat. Mater. 1, 411 (1992).

${ }^{9}$ C. Wild, R. Kohl, N. Herres, W. Muller-Sebert, and P. Koidl, Diamond Relat. Mater. 3, 373 (1994).

${ }^{10}$ A. N. Kolmogorov, Dokl. Akad. Nauk 65, 681 (1949).

${ }^{11}$ A. V. D. Drift, Philips Res. Rep. 22, 267 (1967).

${ }^{12}$ F. Paritosh, D. J. Srolovitz, C. Battaile, X. Li, and J. E. Butler, Acta Mater. 47, 2269 (1999).

${ }^{13}$ S. Barrat, P. Pigeat, and E. Bauer-Grosse, Diamond Relat. Mater. 5, 276 (1996).

${ }^{14}$ D. W. Shaw, J. Cryst. Growth 47, 509 (1979).

${ }^{15}$ Y. V. Kaenel, J. Stiegler, E. Blank, O. Chauvet, Ch. Hellwig, and K. Plamann, Phys. Status Solidi A 154, 219 (1996).

${ }^{16}$ C. C. Battaile, D. J. Srolovitz, and J. E. Butler, J. Mater. Res. 14, 3439 (1999).

${ }^{17}$ P. K. Bachmann, W. Eberhardt, B. Kessler, H. Lade, K. Radermacher, D. U. Weicher, and H. Wilson, Diamond Relat. Mater. 5, 1378 (1996). 